«Responsabilité et énonciation dans la presse quotidienne : questionnements sur les observables et les catégories d'analyse ", dans $S E M E N$ 22, Presses universitaires de Franche-Comté, novembre 2006, p. 45-59.

\author{
Sophie MOIRAND \\ EA SYLED-CEDISCOR \\ Université Sorbonne nouvelle - Paris 3 \\ Version provisoire
}

\title{
Responsabilité et énonciation dans la presse quotidienne : questionnements sur les observables et les catégories d'analyse
}

Un certain nombre de questions ont surgi lorsque j'ai voulu mettre à l'épreuve des notions d'énonciation et de responsabilité les analyses que j'avais effectuées ces dernières années sur la presse. J'ai alors essayé d'envisager « les problématiques » ou « les conceptions » énonciatives (Fuchs 1981, Delesalle 1986) du point de vue d'une analyse du discours mise à l'épreuve de données empiriques 1 , davantage que du point de vue des théories du langage, vers lesquelles elles semblent tendre dans les textes d'origine (de Bally à Benveniste et à Culioli, de Austin à Grice et à Ducrot, chez Volochinov et Bakhtine). Je commencerai par quelques questionnements sur les notions de responsabilité et d'énonciation, avant de proposer un «modèle dialogique » de l'énonciation que je mettrai à l'épreuve de données empiriques, ici des unités discursives empruntées à la presse française, la responsabilité dans une approche linguistique du discours des médias étant pour moi à rapporter aux choix (conscients ou inconscients) de l'auteur dans sa façon de "nommer » les acteurs et les actions et dans celle de « représenter » le discours des autres.

\section{Confronter les médias aux notions de responsabilité et d'énonciation}

Confronter les médias aux notions de responsabilité et d'énonciation conduit à s'interroger sur le lien supposé que le «et» introduit entre elles deux. On se contentera de les rapporter aux contraintes socio-institutionnelles et médiologiques de la presse quotidienne.

Il s'agit en effet d'observer une pratique langagière professionnelle particulière, constituée de genres "seconds", au sens de Bakhtine, en tous cas de genres « construits », qui s'inscrivent dans une institution qu'on peut considérer comme une " communauté langagière », elle-même inscrite dans un "monde social » particulier, le monde des médias. Or l'observation d'une page de journal se caractérise par une hétérogénéité manifeste à plusieurs niveaux, sémiotique, textuelle, scripturale, qui pose, avant toute analyse énonciative, la question de la dilution, ou de l'éclatement, ou de la superposition de responsabilités éditoriales collectives.

\subsection{Du côté de la responsabilité}

Notion absente en entrée des dictionnaires de sciences du langage, la responsabilité apparaît dans les articles sur "l'énonciateur » et fonctionne comme un équivalent de « la prise en charge énonciative » dans certains articles sur l'énonciation.

Mais la responsabilité est plutôt une notion philosophique, en partie liée à la morale et, pour ce qui est de l'éthique des pratiques langagières médiatiques, à ce qu'on est en droit d'attendre des professionnels du langage que sont les journalistes, c'est-àdire, comme le disait Gardin en 1999, « un comportement langagier autre qu'instinctif » (Gardin 1999 : 61). Pour donner un exemple, si on peut éventuellement

${ }^{1}$ Je remercie Raphël Micheli pour ses remarques judicieuses, qui m'ont permis de mieux argumenter ma position. 
accepter qu'un citoyen ordinaire parle d' « otages » à propos des usagers empêchés de voyager en raison de mouvements sociaux, il est pour le moins surprenant d'entendre ce mot repris (sans «distance») par des professionnels du langage au moment même où d'autres journalistes sont réellement retenus en otage en Irak (Moirand et Porquier 2006). En ce sens, c'est à travers les opérations de référence que la responsabilité, ici professionnelle, me paraît liée à l'énonciation.

La page de journal est par ailleurs constituée d'unités discursives, rédigées par des scripteurs différents, dans des lieux et à des moments différents, accompagnées de photographies ou d'infographies ad-hoc ou empruntées à des banques de données. Bien souvent l'auteur d'une de ces unités discursives (le correspondant local, le reporter à l'étranger, le journaliste free-lance...) ne sait pas en compagnie de quelles autres unités discursives son texte sera publié, ni quels éléments paratextuels apparaîtront avec son texte. La responsabilité n'est-elle pas alors éditoriale ? Du côté donc des rédacteurs en chef, ou des différentes rédactions, qui décident, et des contraintes médiologiques du support. Il y a cependant, surplombant la diversité des intervenants et la diversité des scripteurs, une responsabilité singulière, celle de l'instance socio-institutionnelle du journal (y compris aujourd'hui dans ses relations aux actionnaires - voir par exemple Rimbert 2005) et c'est d'ailleurs cette responsabilité-là que l'on peut traduire en justice et qu'on met en cause : celle de l'éditeur, du journal, du copyright.

La question que pose la relation entre la responsabilité et l'énonciation est d'ordre différent : cette responsabilité éditoriale globale se répercute-t-elle dans la prise en charge par un auteur singulier d'une unité discursive présente sur l'aire de la page ? ou plutôt comment l'instance socio-institutionnelle intervient-elle auprès de chacune des instances singulières qui se manifestent dans l'espace d'une page ou d'un numéro ? En tant que linguiste, dispose-t-on de catégories qui permettent de dégager les « observables » de cette responsabilité-là?

Dans la matérialité des surfaces discursives, il arrive que l'unité de l'instance globale s'efface devant la présence de références (nominations, autodésignations...) à la diversité des auteurs ou des locuteurs présents sur une même page, et qui peuvent rendre compte ainsi de la complexité du cadre participatif et/ou énonciatif ${ }^{2}$ :

- Il y a des articles signés et d'autres non signés, et parmi les articles signés des journalistes qui ont des statuts différents (envoyé spécial, correspondant, etc.), et un certain nombre d'articles écrits par des non-professionnels à qui l'on réserve des rubriques spéciales (pages Opinions ou Débats ou Courrier des lecteurs, etc.), dans ce $\mathrm{qu}^{\prime}$ on appelle parfois, non sans ironie, les pages « démocratiques » (Martin-Castelnau 2005). La responsabilité est ici auctoriale, voir éditoriale pour certains responsables du journal, d'une rubrique ou d'un cahier spécial.

- Il suffit de balayer du regard une seule page pour repérer nombre d'énoncés représentés (outre les interviews): des énoncés cités, rapportés, mentionnés, imaginés dans les bulles des dessins de presse, énoncés typographiquement repérables et qui font intervenir les voix d'autres communautés, jusqu'à construire cette «texture énonciative» particulière de certaines articles: un «intertexte plurilogal », faits de discours plus ou moins « situés ».

Il y a donc un éclatement des «instances responsables » des dires produits ou convoqués, certains y voient une hiérarchie, d'autres une dilution des responsabilités, même s'il y a une instance unificatrice qui délègue l'instance

2 Les lecteurs de la presse semblent attacher peu d'importance aux signatures, si l'on compare à la connaissance qu'ils ont des présentateurs ou des reporters des journaux télévisés, davantage "médiatisés » et même "peoplelisés ». Ainsi, une bonne partie des lecteurs de Libération ont découvert le nom de Florence Aubenas lorsqu'elle a été prise en otages en Irak alors qu'elle avait déjà signé de nombreux reportages de son nom. 
éditoriale aux responsables des rubriques ou des cahiers. Ce qui ne veut pas dire que ces différentes instances contrôlent ou assument totalement la "prise en charge énonciative » de chacune des unités discursives, voire des titres qui constituent des unités à part entières, produits souvent par d'autres que les scripteurs des articles qu'ils annoncent (et à un moment et un lieu autres).

\subsection{Du côté de l'énonciation}

« Nous ne connaîtrons jamais que des énonciations énoncées » (Todorov), " Il n'y a pas d'énoncé sans énonciateur » (Culioli) : si l'on est d'accord avec ces assertions, on se demande comment observer les traces de l'énonciateur ou de l'énonciation. Or la question des «observables » de l'énonciation ne se pose pas de la même façon selon qu'on s'interroge sur l'activité de production des énoncés ou sur l'effet recherché sur les interlocuteurs.

On peut concevoir l'énonciation (Dubois 1969: 100), comme «le surgissement du sujet dans l'énoncé »: on fait alors l'hypothèse que l'énonciation laisse des traces, des indices, des marques dans l'énoncé. On fonctionne ainsi sur du "discontinu », dit Jean Dubois. Toutes les catégories de langue, qui s'avèrent des observables de l'énonciation, sont en effet des unités discrètes. Elles permettent d'observer «la distance » mise par le locuteur entre lui-même et ce qu'il dit au niveau local... Mais, poursuit Dubois, "il n'existe pas de moyens privilégiés, de classes de mots particulières pour signifier cette distance; telle structure repérée se révèle l'instant suivant avoir une autre signification. La distance trouve la meilleure expression dans le contour d'intonation qu'on ne peut réduire à des unités discrètes » (ibidem, p. 104). À défaut d'intonation qu'on ne peut repérer en tant que telle dans l'ordre écrit, c'est la notion d' «éclairage », empruntée à la conception de l'argumentation de JeanBlaise Grize, qui m'a paru la plus proche du continu de l'intonation, parce qu'elle permet de saisir « le ton » de l'énoncé (voir infra).

Si on présente l'énonciation comme la relation que le locuteur entretient avec son interlocuteur (les observables sont alors, entre autres, les modalités intersujets ou modalités pragmatiques), on peut basculer du côté des conceptions du langage comme action. On s'interroge sur les fonctions des énoncés, qu'on peut observer aussi bien au niveau local (micro-actes de langage) qu' au niveau global (macro-actes ou visées pragmatiques). Mais l'analyse des énoncés de la presse, unités discursives majoritairement "longues », conduit à privilégier les fonctions globales du texte au détriment du local, fonctions étroitement dépendantes des fonctions sociales des médias: informer, rapporter, commenter, voire conseiller ou expliquer et, à un autre niveau, essentiellement sociologique, divertir ou séduire.

On peut cependant se mettre d'accord, il me semble, sur ce qui relève des études de l'énonciation, comme le propose Simone Delesalle (1986:7) :

Seront ainsi évoqués les éléments proprement linguistiques, discrets ou non, qui ne peuvent être compris que par référence au plan énonciatif : le problème du sujet de l'énonciation, le rôle de l'interlocuteur, et le rapport de l'énonciateur à son énoncé ; et ce dans une prise en charge historicisée de phénomènes tels que les actes de langage, les indexicaux, la construction de la référenciation, la performativité, le dialogisme sous ses différentes formes ou l'hétérogénéité du discours.

Face à cette diversité de catégories (grammaticales, pragmatiques, sémantiques), on repartira de la question des observables à partir de données empiriques, parce que la question qui se pose, en analyse du discours, c'est celle de la relation entre des catégories qui permettent des observations « locales » et le flux continu du discours, cette énonciation actualisée qui est «ce qui émerge d'un océan sans limites, le discours intérieur» (Bakhtine), et qui inscrit les fils verticaux des discours 
transverses, discours antérieurs ou à venir, produits ailleurs et par d'autres, dans le fil horizontal que suit l'ordre du texte (Moirand 2005). Car mettre l'énonciation à l'épreuve des données empiriques, c'est ici la rapporter à l'orientation argumentative de l'ordre du texte, puis à l'espace de la page ou du numéro. C'est également « penser » le fonctionnement discursif de l'énonciation à travers la distribution et la combinaison des traces des opérations langagières dans le fil du texte et à travers la diversité des discours qui le traversent, et qui concourent à une rhétorique des genres.

Un dialogisme "pensé » entre problématiques énonciatives et théories discursives me paraît donner un ancrage théorique aux observations rendant compte des relations entre énonciation et responsabilité. Pour plusieurs raisons que je résume rapidement ici :

- Ce ne sont pas les interlocuteurs qui interagissent directement dans la presse, mais les textes, les énoncés, les mots eux-mêmes, les titres, les photos, les dessins de presse, avec les discours qu'ils transportent, ceux qu'ils anticipent et ceux qu'ils rencontrent sur l'aire de la page... Les discours des médias sont essentiellement des discours « médiateurs » d'autres discours.

- Ce n'est pas la structure de l'énoncé qui importe, ni même le (ou les) sujet(s) énonciateur(s) qui s'effac(ent) et se montr(ent), non plus les relations interpersonnelles entre les co-énonciateurs ou les acteurs, mais les relations interactionnelles entre les discours qui s'énoncent et se rencontrent, sans l'avoir voulu, et qui, en même temps, énoncent leurs rapports aux discours autres.

L'observation intègre donc, au niveau local, les traces des opérations de référence, de prédication et d'énonciation, et prend en compte, au niveau global, le fil du discours avec ses caractéristiques intratextuelles, comme la transformation des objets de discours selon "l'éclairage » qu'on leur donne (Grize 1992, 2005), et ses caractéristiques intertextuelles (la distance " marquée » avec des discours " situés ») et interdiscursives (celles qui renvoient à l'histoire et à la mémoire). On fait l'hypothèse que ces traces plus ou moins marquées d'interactions discursives participent à l'orientation rhétorique de l'article comme de la page de journal et parfois du numéro, voire d'une suite de numéros, et que la responsabilité interviendrait dans la représentation que l'on « donne à voir » de ces interactions.

\section{Un modèle dialogique de l'énonciation confronté à des unités empiriques}

Ce qui caractérise à première vue l'inscription du scripteur professionnel dans la presse, c'est l'absence d'un «je », qui manifesterait une coïncidence entre sujet de l'énoncé et sujet de l'énonciation. C'est également la rareté des formes d'interrogation ou d'intimation, pour reprendre les termes de Benveniste, excepté dans les textes et surtout les titres, les sous-titres ou les intertitres à coloration didactique ou dans les textes de conseil. Les formes d'énoncés les plus fréquentes sont des assertions, accompagnées ou non de modalités et de marques de distance, qui rendent compte de ce qu'on a vu, de ce qu'on sait, de ce qu'on a appris par d'autres, d'où la présence constante de discours « autre » et une responsabilité qui se manifeste dans l'usage que l'on en fait (consciemment ou non). À des fins d'interrogation sur les notions de responsabilité et d'énonciation, on propose de confronter le modèle proposé supra à plusieurs facettes de la page de journal.

\subsection{L'espace d'une unité discursive singulière}

On rapporte la distribution des traces, indices ou marques, forcément discontinue, à l'orientation pragmatique globale, qui se manifeste au travers de "l'éclairage» construit au fil du texte. 
Dans une brève, au hasard des pages de Libération

Ex. 1:

Agriculture

Deux parcelles d'OGM détruites en Auvergne

Dans la nuit de lundi à mardi, des membres du collectif des Faucheurs volontaires ont détruit deux parcelles de maïs transgéniques dans le Puy-de-Dôme, département le plus génétiquement modifié de France avec une vingtaine d'hectares. Exploités par le laboratoire Meristem Therapeutics, ces plants synthétisent des lipases gastriques, médicaments destinés à soulager les malades atteints de muciviscidose.

[Libération 03.08.05]

Il s'agit d'une brève, dont on ne sait à qui attribuer la paternité. Elle se présente comme une suite d'assertions relatant des faits que le scripteur assume comme des realia (sans distance). Mais s'il y a effacement des actants dans le titre, il y a repositionnement de ces actants en position sujet dans le texte, ce qui permet de désigner les acteurs de cette destruction et de faire appel à des discours déjà emmagasinés en mémoire à propos des OGM, des destructions de plants transgéniques et des «faucheurs » de ces plants.

Deux traces de l'éclairage que le scripteur anonyme construit me paraissent pourtant manifester son intervention, ces éléments n'étant pas nécessaires à la relation des faits :

- le plus génétiquement modifié (a)

- destinés à soulager les malades (b)

Si on s'attache à la caractérisation apportée au Puy-de-Dôme (a), on peut y trouver la raison de la destruction. Mais si on s'attache à la caractérisation apportée aux médicaments (b) et indirectement aux plants transgéniques, on construit un lien entre les deux phrases de cette brève : pourquoi détruire un maïs cultivé pour une «bonne cause »? On retrouve ici des arguments de discours circulants à propos des OGM, que le scripteur rappelle dans les caractérisations qu'il donne, parce que, comme le dit Grize (2005 : 42): «Les objets du discours doivent être éclairés, ce qui revient à mettre en évidence quelques-unes de leurs facettes et à en occulter d'autres et tout éclairage colore ce qu'il illumine, ce qui découle du fait qu'il se sert des préconstruits culturels qui ne sont jamais neutres». Or, l'éclairage que donne le scripteur repose sur le choix des mots et de la syntaxe lors des opérations de référence et de prédication (les déterminations), et pas seulement sur les traces des opérations dites d'énonciation (ici l'assertion et l'accompli, qui marquent la prise en charge sous-jacente du scripteur - « je vous dis que » - et la réalité des faits relatés).

Dans des titres et des sous-titres, au hasard du balayage des pages

Ex. 23:

- Plus de 1000 hectares de maïs transgénique exploités dans 12 départements

OGM : du maïs déjà cultivé en France à grande échelle [p. 1]

- AGRICULTURE Dans le plus grand secret, des cultures de maïs transgéniques ont été plantées dans le Sud-Ouest.

OGM : déjà un millier d'hectares en France [p. 14]

[le Figaro 06.09.05]

- Le gouvernement a caché l'existence des cultures commerciales d'OGM

La révélation que 1000 hectares de maïs transgénique étaient cultivées en France a déclenché de vives réactions chez les élus du Sud-Ouest.

[le Monde 09.09.05]

3 Dans les exemples, c'est moi qui souligne en gras. 
Il semble, comme le dit Grize, qu'il s'agit moins ici de communiquer des faits que de donner d'emblée un certain " éclairage » à cette information à travers les marques de la quantification, de l'accompli ou de l'intensif (plus de, le plus grand, déjà), information que le Monde reprendra en utilisant des mots qui renforcent encore "l'éclairage » des titres du Figaro.

C'est là souvent la fonction des titres, qui disent davantage que l'énoncé des faits en raison de ce qu'ils inscrivent des discours antérieurs ou à venir et de l'éclairage donné par les mots et la syntaxe lors des opérations de référence (nomination, désignation, caractérisation) :

Ex. 3 :

L'Europe résiste [page 1]

Les Européens disent aussi non aux OGM [p. 2]

[Libération 25.06.05]

La Turquie, une "Chine " à nos portes

Européenne ou pas, la Turquie affole les investisseurs. "Nouvelle Chine» économique, le pays de Kemal Atatürk fait les choux gras d'Oberthur et de ses cartes à puce

[le Journal du Dimanche 10.10.05]

Le drame serait que les oiseaux migrateurs volent vers l'Afrique qui ne dispose d'aucun réseau sanitaire pour contenir le Tchernobyl aviaire

[Paris-Match 20-26.10.05]

Grippe aviaire Un fléau de plus en Afrique

[Liberation 11.02.05]

Derrière une quantification, une désignation qualifiante ou ce que j'ai appelé un " mot-événement » (Tchernobyl), c'est bien l'auteur du titre qui donne un «éclairage » à l'information: dans le premier titre de l'ex.3, aussi rappelle à la mémoire des lecteurs le non au référendum sur le projet de constitution européenne opposé par la France et les Pays-Bas mais les mots résiste, Europe, Européens peuvent également évoquer les controverses entre les États-Unis et l'Europe, entre le modèle ultra-libéral et un modèle social "européen". Caractériser la Turquie comme une "nouvelle Chine ", c'est rappeler à la fois l'intérêt des échanges avec ces pays mais aussi la crainte des importations de produits à bas prix (le textile, par exemple), etc. Parler de « fléau » rappelle la peste, et le segment de plus qu'il y en a d'autres, qui surgissent en mémoire, orientant ainsi l'interprétation.

L'éclairage repose donc pour moi sur "la mémoire des mots », qui, comme le dit Bakhtine, " n'oublient jamais leur trajet » et transportent avec eux les discours qu'ils ont traversés, sans que le scripteur en soit toujours conscient, dans le fil horizontal du discours: "En résumé, les phénomènes d'éclairage apparaissent comme le résultat de mécanismes plus ou moins consciemment mis en place et qui doivent conduire le lecteur à inférer par lui-même un jugement de valeur »(Grize 1992 : 25). Du coup, cette perspective fait basculer l'énonciation du côté des destinataires plutôt que du côté du «point de vue» du locuteur : si l'on est d'accord avec l'approche praxématique sur le caractère « historicisé, culturalisé, socialisé » du " dialogisme de la nomination ", que l'on peut rapporter au point de vue (Détrie, Siblot, Vérine éds 2001 : 255), il reste qu'une part des valeurs auxquelles renvoie l'énonciation dans la presse échappe partiellement au scripteur et renvoie à la responsabilité du lecteur.

\section{Dans un article d'information}

Le texte qui suit a paru dans une double page de Libération annoncée à la une par le titre déjà cité dans l'ex. 4 : Un fléau de plus en Afrique. La mention, "Lagos, de notre correspondante» révèle, en début d'article et en dessous du titre surplombant cet article et une photo, le lieu du reportage sur l'arrivée de la grippe aviaire en Afrique : 
Ex. 4 :

Lagos de notre correspondante

Les autorités nigérianes appellent à l'aide: "Laboratoires, vaccins, soutien technique et échanges avec les pays confrontés à la même expérience », a expliqué le ministre de l'Agriculture, Adamu Bello. En clair, un soutien logistique massif pour lutter contre l'épidémie de grippe aviaire qui est en train de décimer les gallinacés dans le nord du pays.

"La majorité des pays veut aider, mais il faut leur donner une liste de besoins précis, et ça n'a pas été le cas", déplore toutefois une source diplomatique. Les Etats-Unis ont déjà promis 20 millions de dollars et un laboratoire, et trois experts de la FAO sont attendus pour aider à définir les besoins dans les zones infectées. Des épidémiologistes de I'OMS sont également en route.

Frontières fermées. "Dans ce genre de situation, le dispositif international se met très rapidement en place ", souligne un bailleur de fonds. Mais la grippe aviaire ne perd pas de temps non plus. Un foyer a été découvert mercredi à Kaduna, dans le nord du pays ; le lendemain, le virus H5N1 était détecté dans deux autres Etats proches, Kano et Plateau. Une trentaine de fermes seraient déjà infectées et au moins 100000 volailles sont mortes. Les pays voisins du Nigeria ont décrété l'interdiction de l'importation de volailles en provenance de ce pays. Pour le moment, aucun cas humain n'a été signalé, mais deux enfants malades d'une ferme de Kaduna ont subi des examens médicaux. Selon les autorités, des quarantaines ont été mises en place autour des fermes suspectes. Un témoin rapporte qu'il $n^{\prime} y$ a pas de dispositif visible sur la route entre Abuja, la capitale, et Kaduna. "Les poulets se promènent en liberté, la grippe aviaire peut être partout et nulle part. » A Kano, les villageois brûlent des poulets morts sans aucune précaution.

Le nord du Nigéria est rural, densément peuplé et très pauvre. C'est dans cette zone que les taux de scolarisation sont les plus bas. Le taux de couverture vaccinale y est inférieur à celui du sud du pays, selon une étude datée de 2003, il n'atteint même pas $1 \%$ dans certains Etats comme le Jigawa, frontalier de Kano. Dans cette région, des leaders religieux musulmans ont lancé en 2003 une campagne dénonçant la vaccination contre la poliomyélite, accusée d'être une invention des Américains pour stériliser les musulmans. La vaccination a été interrompue pendant un an et demi. A partir du Nigeria, plusieurs pays qui avaient éradiqué la polio ont été recontaminés.

Messages simples. De cette crise est né un fort réseau local susceptible aujourd'hui d'aider à diffuser l'information sur la grippe aviaire. "Il y a des relais dans chaque Etat, et le réseau des chefs traditionnels fonctionne très bien ", explique la porte-parole de l'Unicef au Nigeria. Les messages sont simples: respecter les règles d'hygiène de base et éloigner les enfants des poulets.

La FAO a par ailleurs demandé la fermeture des marchés de volailles dans les zones infectées. Craignant des pertes financières, les éleveurs continuent en effet d'écouler en hâte leurs poulets morts pour échapper aux mesures de quarantaine. Le gouvernement nigérian a certes annoncé des mesures de compensation financière, mais la prudence est de mise. Convaincre les éleveurs de renoncer à leur source de revenus ne sera pas chose facile. Business oblige, les volailles risquent de passer à travers les mailles du filet. VIRGINIE GOMEZ

[Libération 11.02.06]

Article envoyé de l'étranger, il est constitué de dires cités en italiques et entre guillemets, empruntés à différents acteurs (le ministre de l'agriculture, une source diplomatique, un bailleur de fonds, un témoin, la porte-parole de l'Unicef au Nigéria...) et de dires reformulés ou d'actes de langage rapportés (les autorités nigérianes appellent à l'aide, la FAO a par ailleurs demandé la fermeture..., le gouvernement nigérian a annoncé des mesures...). Mais les faits rapportés sont également issus de dires recueillis (médias locaux, agences locales, témoins, etc.), parfois sans distance (donc assumés formellement comme « vrais ») :

Un foyer a été découvert mercredi à Kaduna, dans le nord du pays

le lendemain, le virus H5N1 était détecté dans deux autres États proches

avec, pour certains, des marques de « distance » explicites :

une trentaine de fermes seraient déjà infectées

Selon les autorités, des quarantaines ont été mises en place

un témoin rapporte qu'il n'y a pas de dispositif visible 
Si l'on trouve peu de traces explicites de la présence de l'auteur, celui-ci a néanmoins la responsabilité du choix des informations, de la mise en scène de cet intertexte plurilogal caractéristique du genre, avec ses contraintes de temps et d'espace. L'éthique de la profession et la pratique professionnelle conduisent le scripteur à montrer «la distance» ressentie entre son énoncé et les dires qu'il recueille (dans une autre langue) et qu'il rapporte. L'auteur est bien là, et pas seulement au travers des quelques traces qui manifestent sa présence, telles «en clair », lorsqu'il reformule les propos du ministre, ou « déplore », lorsqu'il interprète l'attitude d'une «source diplomatique » dont il rapporte les dires, ou «selon » pour marquer sa distance envers ce qu'il a appris ou ce qu'on lui a dit. Il est constamment là, même lorsque la distance tend vers zéro lorsque le fait est donné sans réserve, à l'accompli ou avec une forme actualisée de l'accomplissement (et qu'on note une absence de tension - Dubois 1969: 109), ce qui veut dire justement qu'il assume pleinement la réalité $\mathrm{du}$ fait en question. Il est donc toujours présent, à travers la façon dont il désigne et énonce les faits, la façon dont il représente les dires, la façon dont il «éclaire» les informations sur le Nigéria pour les lecteurs français, ce qui oriente le fil du texte vers la chute finale, justifiant ainsi le titre et son sous-titre (sans doute conçus à Paris, par les responsables de la double page) :

La grippe aviaire menace l'Afrique

Touchant le continent le moins armé sanitairement, les cas signalés sont alarmants

On peut opposer à cette forme d'inscription de discours autre, typique des textes de reportage, une autre forme d'hétérogénéité, de l'ordre du suggéré ou du masqué, $\mathrm{qu}^{\prime}$ on trouve plutôt dans les chroniques, les commentaires, les dessins de presse, les éditoriaux, genres qui tendent vers une énonciation davantage subjectivisée.

\section{Dans un éditorial}

Il s'agit là d'un genre, autodésigné et généralement signé, dans lequel on s'attend à retrouver des traces du surgissement du sujet de l'énonciation dans l'énoncé. Mais, au-delà de «l'énonciation énoncée » (Coquet 1983), c'est dans la façon de présenter, de nommer et d'éclairer les faits et les dires tels qu'il les perçoit et qu'il les rapporte, et dans la façon dont il les "montre », que l'énonciateur se glisse, davantage encore qu'au travers des marques de la personne ou des modalités appréciatives, considérées souvent comme prototypiques des traces de subjectivité.

Ex. 5 :

Marge d'incertitude Par Dominique Quinio

Mission vraiment impossible. Informer sans affoler. Avertir sans semer la panique. Expliquer qu'on ne sait pas tout, sans donner l'impression de ne rien maîtriser. En dire trop, ne pas en dire assez. L'exercice imposé par l'avancée de la grippe aviaire en Europe aux autorités sanitaires et politiques tient de l'équilibrisme. Parce que le risque existe, certes, mais que le pire n'est pas sûr et que ce «pire » peut se produire lors des prochaines migrations d'oiseaux ou dans dix ans. " $C^{\prime}$ 'est un phénomène naturel et il viendra ", a ainsi analysé avec un flegme tout britannique le directeur général de la santé du Royaume Uni qui a chiffré le nombre des victimes potentielles à 50000 morts!

Les crises sanitaires se succèdent. Il y eut la vache folle et la fièvre aphteuse. Aujourd'hui ce sont les oiseaux migrateurs et leurs frères domestiques qui portent la menace: le virus animal qui, s'il se combinait avec un virus humain, pourrait provoquer une épidémie redoutable, comparable à la grippe espagnole au début du siècle dernier. La Roumanie, la Turquie sont touchées. L'Europe hausse le niveau de se alertes. Les procédures se mettent en place. Et on le fait savoir...

Car les responsables politiques ont bien compris, que si survenait la catastrophe, ils seraient vite soumis au feu des critiques et rappelés à leurs responsabilités. Y compris en justice, comme l'ont prouvé les scandales du sang contaminé et aujourd'hui le 
dossier de l'amiante. Alors le principe de précaution se met en œuvre sur toute cette partie de la planète qui peut anticiper, et tenter de prévenir, les catastrophes qui l'atteignent. Les autres regardent les épidémies bien réelles, comme celle du sida, continuer leurs ravages. Ou les blessés du tremblement de terre au Pakistan mourir de ne pas être soigné à temps...

Pour l'heure, les populations gardent leur sang-froid même si, selon un sondage français, elles se sentent mal informées. Comment pourrait-il en être autrement? Personne n'est en mesure de délivrer une information irréfutable, celle qui rassurerait totalement ou alarmerait à coup sûr. L'avenir ne peut être prédit qu'au conditionnel. L'incertitude, principe de réalité.

[La Croix 17.10. 05]

L'auteur rapporte et commente les actes de langage des autorités politiques et sanitaires: informer, affoler, avertir, dire, faire savoir... Une seule citation, dont l'encadrement se termine sur un point d'exclamation : il s'agit donc d'allusions aux formes de dire qui circulent et aux difficultés à dire face aux exigences des citoyens des pays développés (le feu des critiques, le rappel des responsabilités). La présence de l'énonciateur est moins dans les traces locales d'opérations énonciatives «discontinues" que dans l'organisation de la schématisation que le discours construit au fil du texte, ce qui est typique des éditoriaux.

Autre caractéristique du genre, c'est la présence ici d'allusions à d'autres événements, qui fonctionnent comme autant de rappels mémoriels à destination des lecteurs et participent également de ce fait à l'orientation pragmatique construite au fil du texte: la vache folle, la fièvre aphteuse ne désignent pas ici les maladies mais deviennent des «mots-événements » qui rappellent les crises politico-scientifiques qu'elles ont déclenchées. Il en est de même du sang contaminé et de l'amiante. Et le rappel ne s'étend pas seulement aux événements récents: la grippe espagnole fait partie d'une mémoire collective (Halbwachs 1950). Ainsi, plusieurs domaines de mémoire sont convoqués, qui renvoient aux savoirs et à l'histoire, récente ou plus ancienne, mais également aux discours qui ont été tenus au moment de ces événements, en particulier, pour les plus récents, par les autorités sanitaires, politiques, économiques, etc.

Ces rappels contribuent à l'éclairage que le scripteur donne : il prend le lecteur à témoin, il contribue ainsi à la construction des opinions à travers le micro-univers qu'il propose, mais dont l'effet pragmatique dépend des mémorisations que les mots évoquent, et qui donc échappent partiellement à l'auteur du texte.

Ainsi, si l'on peut observer des différences entre les unités discursives réparties sur l'aire de la page de journal, c'est dans les stratégies d'éclairage proposées au lecteur telles qu'elles se distribuent et se manifestent au fil du texte (au niveau global) plutôt que dans la présence plus ou moins grande de l'énonciateur (au niveau local). C'est donc dans les rappels des faits, des dires, des discours autres, et dans la façon de les organiser textuellement, que s'exercerait la responsabilité du scripteur, tout en sachant qu'une part de la rhétorique lui échappe, puisqu'elle réside dans ce que les mots, les dires, les rappels évoquent pour chacun des lecteurs, et donc différemment de l'un à l'autre. Mais la responsabilité "singulière" d'un auteur, par ailleurs infléchie par les normes du genre, par le ton du journal, et par l'intériorisation de contraintes socio-institutionnelles, l'est aussi par la présence d'autres textes sur l'aire de la page et au fil des numéros qui se suivent à propos d'un événement.

\subsection{L'espace-temps de la page ou de l'événement}

Les contextes spatio-textuels, spécifiques de la presse écrite, construisent des interactions textuelles qui semblent échapper aux auteurs des unités discursives ainsi rassemblées et aux responsables éditoriaux des rubriques, des cahiers et des 
quotidiens eux-mêmes: c'est la lecture, rarement exhaustive, souvent de type sélective ou "balayage » (Moirand 2006a), qui assume la responsabilité des échos formels et sémantiques d'un texte à l'autre d'une même page et donc les mises en relation spatio-textuelles et intertextuelles, a fortiori les relations interdiscursives qui relèvent de la mémoire.

De plus, cette spécificité s'inscrit dans un contexte temporel particulier à la presse quotidienne, soumise à «la tyrannie de l'instant» (Wolton). La mise en page peut changer au dernier moment, personne n'a le temps de relire en détail les informations qui arrivent au gré des dépêches d'agences, des envois de dernière minute (par satellite) des reporters sur le terrain - toutes chose qui « bousculent » la production jusqu'au dernier moment - ni de s'interroger vraiment sur les effets construits par la co-présence de certains textes ou documents sur l'aire de la page, comme le fait un chercheur universitaire. Encore faut-il ici différencier les types de pages qu'on choisit d'analyser.

Aux pages qui construisent une véritable mise en scène d'un fait ou d'un événement (voire une hyperstructure : Adam et Lugrin ici même et Moirand 2006a), on peut opposer les pages spécialisées intitulées «débats » ou «opinions » des grands quotidiens nationaux dans le monde, pages qui réunissent des genres comparables dont les thèmes peuvent être très disparates et dont les auteurs ne sont pas forcément des professionnels des médias.

Les pages "événement », " fait du jour », " actualités », souvent annoncées à la une par un titre qui éclaire leur orientation (Grippe aviaire/Tout savoir sur la menace ou Grippe aviaire/ Un fléau de plus en Afrique), correspondent au traitement d'un moment discursif particulier qui généralement s'étale sur plusieurs numéros, voire sur plusieurs semaines ou plusieurs mois, et qui revient cycliquement au cours du temps : ainsi, en France, la grippe aviaire a été l'objet de ce genre de pages en octobre 2005, est revenue à la une en janvier-février 2006 lors de la progression du virus vers l'Europe et de la découverte du premier cas en France (au point d'éclipser les premières manifestations étudiantes) avant d'être écartée de la une et de ces pages événements par les grèves dans les universités.

Lorsqu'on réunit des corpus ayant trait à des moments discursifs particuliers, parce que l'objectif est non pas d'étudier les formes d'énonciation dans la presse mais la façon dont les discours traitent un événement particulier, on est contraint de constituer des corpus suffisamment étendus des différents quotidiens nationaux pour ne pas se faire enfermer par une étude de cas singulière, qui n'est qu' un instant discursif dans la durée d'un événement, et qui ne traite bien souvent qu'une des «facettes» de l'événement. C'est pourquoi le questionnement qui guide l'analyse tient compte alors de la durée du traitement de l'événement.

Il s'avère alors difficile de distinguer, comme on peut le faire sur des corpus de textes énonciativement homogènes et/ou singuliers, des phénomènes particuliers de responsabilité énonciative, qui relèveraient de la sur- ou de la sous- énonciation, comme Rabatel peut le proposer ici même (voir également Rabatel éd. 2004). Ils se distribuent en effet de manière éclatée, diluée, mais souvent complémentaire dans la durée de l'événement, au fil des doubles pages qui lui sont consacrées, mais pas toujours au même moment d'un quotidien à un autre: au bout du compte le traitement énonciatif me semble globalement identique dans la presse actuelle des sociétés démocratiques développées, ce qu'il faudrait vérifier par des études statistiques (Schepens et Viprey ici même).

Les pages « débats » ou « opinions » ne présentent pas la même hétérogénéité, et on observe souvent une grande similarité énonciative et pragmatique d'un texte à un 
autre. Constituées de textes à énonciation subjectivisée (chroniques, commentaires, éditoriaux, dessins de presse, interviews, lettre de lecteurs), elles ont une autre particularité, celle de faire appel, au moins partiellement, à des non-professionnels des médias. La question est alors celle du choix fait par le journal : on voudrait croire que tout le monde peut écrire, mais bien évidemment il n'en est rien. Comme le dit le titre de l'article de David Martin-Castelnau (2004), il y a des «professionnels » de la tribune libre!

Le questionnement qui guide l'analyse essaie de prendre en compte cette hétérogénéité scripturale : qui écrit? au nom de qui ? qui est sélectionné ? par qui ? Là encore se pose la question des observables et des catégories d'analyse: si l'orientation pragmatique ou argumentative de ces textes peut être étudiée à partir des indices formels et sémantiques de l'énonciation ou des traces des interactions textuelles et discursives, peut-on s'engager sur une étude de la responsabilité sans recourir au fonctionnement socio-institutionnel du média, aux critères de sélection des textes et des experts à qui l'on « commande » des papiers ?

Invitée par l'argument du colloque à l'origine de ce numéro à réfléchir sur les notions d'énonciation et responsabilité dans les médias, j'ai rencontré un certain nombre de difficultés à les mettre à l'épreuve d'une analyse de données empiriques. On ne peut en effet assimiler la responsabilité à la prise en charge énonciative, que l'on souscrive à une conception de la communication qui voit dans la responsabilité une notion morale, n'ayant qu'un rapport lointain avec les formes des matérialités discursives (Prodomme 2004, par exemple, sur les discours sur l'éthique des journalistes), ou que l'on souscrive, c'est mon cas, à une conception du discours, proche de celle de Pêcheux, pour qui le locuteur n'est pas la source du sens, le sens se construisant dans l'histoire à travers le travail de la mémoire (Maldidier 1990). Mais on peut en revanche s'interroger sur une éthique langagière, proche de celle proposée par Bernard Gardin.

Si on pense en effet que le langage a une responsabilité dans la construction de la réalité sociale (Searle 1998), cette éthique se manifesterait dans la façon de nommer (désigner, caractériser) les faits et les événements, les acteurs, leurs actions et leurs actes de langage, et dans la façon de représenter leurs dires: une éthique de la responsabilité implique en effet qu'on n'a pas le droit de se désintéresser des conséquences de ses actes de langage, et qu'il n'est pas sans conséquence de choisir, par exemple, entre jeune et racaille, entre étudiant ou anarchiste pour désigner les acteurs d'un événement ou de rapporter sans distance et/ou sans mention d'origine les énoncés qui circulent à propos d'un fait ou d'un événement (Moirand 2006c). Le choix de certaines désignations comme de certaines formulations ou constructions syntaxiques constituent bien souvent des coups de force pragmatiques en politique ("vieille Europe », «croisade», «émeutes », "comportement de gardes rouges », etc.). Un énonciateur responsable devrait alors ne pas se contenter de répéter mais fournir également les moyens de retrouver "l'épaisseur dialogique » du mot, de la formulation ou de l'énoncé, recherche aujourd'hui facilitée par l'usage « intelligent » qu'on peut faire des moteurs de recherche sur l'internet (Moirand 2006b). 
COQUET J.-Cl. (1983) «L'implicite de l'énonciation », Langages 70, p. 9-14.

DELESALLE, S. éd. (1986) Histoire des Conceptions de l'Énonciation, Histoire, Epistémologie, Langage 8- II.

DÉTRIE C., SIBLOT P. et B. VERINE éds (2001) Termes et concepts pour l'analyse du discours, Paris, Champion.

DUBOIS J. (1969) «Enoncé et énonciation », Langages 13, p. 100-110.

FUCHS, C. (1981) « Les problématiques énonciatives : esquisse d'une présentation historique et critique », DRLAV, revue de linguistique, $\mathrm{n}^{\circ} 25, \mathrm{p}$. 35-60.

GARDIN B. (1999) "Les Morales langagières », entretien effectué par Régine DelamotteLegrand, Le français dans le Monde nºspécial, juillet 1999, p. 58-67.

GRIZE J.-B. (1992) "Éclairage », Un signe parmi d'autres, Hauterive, Editions Gilles Attinger, p. 22-25.

GRIZE J.-B. (2006 [2005]) « Le point de vue de la logique naturelle », Doury M. et S. Moirand éds: L'argumentation aujourd'hui. Positions théoriques en confrontation, Paris, Presses Sorbonne Nouvelle, p. 35-43.

HALBWACHS M. (1997 [1950]) La mémoire collective, Paris, Albin Michel.

MALDIDIER D. (1990) L'inquiétude du discours. Textes de Michel Pêcheux choisis et présentés, Paris, Éditions des Cendres.

MARTIN-CASTELNAU D. (2005) "Enquête sur les professionnels de la "tribune libre" ", Marianne 423-424, p. 90-95.

MOIRAND S. (2004a) «L'impossible clôture des corpus médiatiques », TRANEL 40, p. 71-92.

MOIRAND S. (2004b) «De la nomination au dialogisme : quelques questionnements autour de l'objet de discours et de la mémoire des mots ", Dialogisme et nomination, Université Paul Valéry - Montpellier 3, p. 27-61.

MOIRAND S (2005) «Le dialogisme, entre problématiques énonciatives et théories discursives », Cahiers de praxématique 43, p. 189-220.

MOIRAND S. (2006a, à paraître) «De l'aire de la page à l'hyperstructure et à l'écran : comment lire et analyser la presse quotidienne française ", CAUCE, Revista international de Filología y su Didáctica, Universidad de Sevilla, Espagne.

MOIRAND S. (2006b, à paraître) « Entre discours et mémoire : le dialogisme à l'épreuve de la presse ordinaire », TRANEL.

MOIRAND S. (2006c, à paraître) «Le choc des discours dans la presse française : l'exemple des violences urbaines et des manifestations étudiantes", conférence d'ouverture du Colloque international de la FATFA, Adelaide, Australia, 7-9 juillet.

MOIRAND S. et PORQUIER R., (2006, à paraître), « De l'éthique de la nomination à l'éthique de l'interprétation : autour du mot "otage" et de quelques autres ", volume d'hommage à Bernard Gardin, Publications de l'université de Rouen.

PRODOMME M. (2004) «Le discours sur l'éthique professionnelle des journalistes français : le cas du Syndicat National des Journalistes (1990-2000) », COMMposite, v2004 : http:/ / commposite.org/2004/articles/prodho.html

RABATEL A. éd. (2004) Effacement énonciatif et discours rapportés, Langages 156.

RIMBERT P. (2005) Libération de Sartre à Rothschild, Paris, Raisons d'agir.

SEARLE J.R. (1998, traduction) La construction de la réalité sociale, Paris, Gallimard.

TODOROV T. éd. (1970) : L'énonciation, Langages 17.

VOLOCHINOV V.N. (1981) : «Le discours dans la vie et le discours dans la poésie », «La structure de l'énoncé », Todorov, T. : Mikhaïl Bakhtine, le principe dialogique suivi des Écrits $d u$ cercle de Bakhtine, Paris, Seuil, p. 181-215 et 287-314. 